\title{
SINGULARITIES OF SOLUTIONS TO COMPRESSIBLE EULER EQUATIONS WITH VACUUM
}

\author{
Zhen Lei, Yi Du AND Qingtian Zhang
}

\begin{abstract}
Presented are two results on the formation of finite-time singularities of solutions to the compressible Euler equations in two and three space dimensions for isentropic, polytropic, ideal fluid flows. The initial velocity is assumed to be symmetric and the initial sound speed is required to vanish at the origin. They are smooth in Sobolev space $H^{3}$, but not required to have a compact support. It is shown that the $H^{3}$ norm of the velocity field and the sound speed will blow up in a finite time.
\end{abstract}

\section{Introduction}

Euler equation is one of the most fundamental equations in fluid dynamics. Many interesting fluid dynamic phenomena can be described by the Euler equation (see, for instance, [12]). Recently, singularity formation in fluid mechanics has attracted the attention of a number of researchers; see, for instance, $[2,3,5-8,11]$ and two recent review articles $[1,4]$. For compressible Euler equations of the motion of polytropic ideal fluid flows, Sideris (see [13]) proved, under various settings, several very interesting results on the formation of finite-time singularities to solutions whose initial velocity field has a compact support and initial density is strictly positive and is equal to a positive constant outside the support of the initial velocity field. It is very interesting to investigate the long-time behavior of solutions to compressible Euler equations with initial data containing vacuum states, as has been pointed out in [9].

In this short paper, we prove that solutions to compressible Euler equations will develop finite-time singularities for radially symmetric initial data whose initial velocity field has no compact support and initial density contains vacuum states. In particular, it is shown that the $H^{3}$ norm of the velocity field and the sound speed will blow up in a finite time.

To state our theorems, let us begin with the compressible Euler equations for isentropic, polytropic, ideal fluid flows:

$$
\left\{\begin{array}{l}
\rho_{t}+\nabla \cdot(\rho u)=0, \\
(\rho u)_{t}+\nabla \cdot(\rho u \otimes u)+\nabla p=0,
\end{array}\right.
$$

where $\rho(t, \cdot): \mathbb{R}^{n} \rightarrow \mathbb{R}$ is the scalar mass density, $u(t, \cdot): \mathbb{R}^{n} \rightarrow \mathbb{R}^{n}$ is the velocity field, $p(t, x)$ is the pressure, which is given by the equation of state

$$
p=A \rho^{\gamma} .
$$

Here, $A>0$ is an entropy constant, $\gamma$ is the adiabatic index. For polytropic gases, one has $1<\gamma \leq \frac{5}{3}$.

Received by the editors August 12, 2012.

Key words and phrases. Finite-time singularities, compressible Euler equations, vacuum. 
The compressible Euler equations (1.1) are imposed on the following initial data:

$$
\rho(0, x)=\rho_{0}(r), \quad u(0, x)=\frac{x}{r} v_{0}(r) .
$$

Here and in what follows, we will use

$$
r=|x|, \quad x \in \mathbb{R}^{n},
$$

for notational convenience. Denote the sound speed $c(\rho)$ by

$$
c(\rho)=\sqrt{\frac{\partial p(\rho)}{\partial \rho}}=\sqrt{A \gamma} \rho^{\frac{\gamma-1}{2}} .
$$

Our first result is on the formation of singularities of solutions to the compressible Euler equations in three dimensions:

Theorem 1.1. Assume that $\gamma>1, \rho_{0} \geq 0$ and $\left(c_{0}, u_{0}\right) \in H^{3}\left(\mathbb{R}^{3}\right)$ with $c_{0}=c\left(\rho_{0}\right)$. Moreover, assume that $\rho_{0}$ and $v_{0}$ satisfy

$$
\begin{gathered}
\rho_{0}(0)=0, \\
\int_{\mathbb{R}^{3}} \rho_{0}(r) d x>0,
\end{gathered}
$$

and

$$
-\int_{\mathbb{R}^{3}} \frac{(1+r) \rho_{0} v_{0}}{r^{2} e^{r}} d x \geq \sqrt{\frac{A}{(\gamma+1)(4 \pi)^{\gamma-1}}}\left(\int_{\mathbb{R}^{3}} \frac{\rho_{0}}{r e^{r}} d x\right)^{\frac{\gamma+1}{2}} .
$$

Then the solution $(\rho, u)$ to the compressible Euler equations (1.1) with the initial data (1.3) will develop finite-time singularities.

Our second result is on the formation of singularities of solutions to the compressible Euler equations in two dimensions:

Theorem 1.2. Assume that $\gamma>1, \rho_{0} \geq 0$ and $\left(c_{0}, u_{0}\right) \in H^{3}\left(\mathbb{R}^{2}\right)$ with $c_{0}=c\left(\rho_{0}\right)$. Let $K_{0}(r)$ be the modified Bessel function

$$
K_{0}(r)=\int_{0}^{\infty} e^{-r \cosh t} d t
$$

If the initial data $\rho_{0}$ and $v_{0}$ satisfy (1.5),

$$
\int_{\mathbb{R}^{2}} \rho_{0}(r) d x>0
$$

and

$$
\int_{\mathbb{R}^{2}} \rho_{0}(r) v_{0}(r) K_{0}^{\prime}(r) d x \geq \sqrt{\frac{A}{\gamma+1}} \frac{\left(\int_{\mathbb{R}^{2}} \rho_{0}(r) K_{0}(r) d x\right)^{\frac{\gamma+1}{2}}}{\left(\int_{\mathbb{R}^{2}} K_{0}(r) d x\right)^{\frac{\gamma-1}{2}}},
$$

then the solution $(\rho, u)$ to the compressible Euler equations (1.1) with the initial data (1.3) will develop finite-time singularities. 
Remark 1.3. For a polytropic ideal gas, the adiabatic index $\gamma \in\left(1, \frac{5}{3}\right]$ and hence $\frac{2}{\gamma-1} \geq 3$. Owing to the expression of the sound speed in (1.4), it is easy to get

$$
\rho_{0}=(A \gamma)^{-\frac{1}{\gamma-1}} c_{0}^{\frac{2}{\gamma-1}}
$$

Consequently, one also has $\rho_{0} \in H^{3}\left(\mathbb{R}^{n}\right)$ under the condition that $c_{0} \in H^{3}\left(\mathbb{R}^{n}\right)$, which in turn implies that $\rho(t, \cdot) \in H^{2}\left(\mathbb{R}^{n}\right)$ as long as the solution is smooth. For $\gamma>\frac{5}{3}$, local well-posedness theory in Theorem 2.1 implies that $\rho \in C\left([0, T) \times \mathbb{R}^{n}\right)$.

Remark 1.4. For the non-isentropic, polytropic, ideal gases, the entropy $S$ is transported by the flows. It is easy to verify that our proofs of the blow up parts in Theorems 1.1 and 1.2 are still true. However, the local well-posedness of the coupled system with initial vacuum is much more complicated (see, for instance, [9]). We do not pursue this issue in this short paper.

There are several ingredients in the proofs of the above theorems. The first one is to write the compressible Euler equations (1.1) as a quasi-linear wave-type equation in terms of $\rho$ with inhomogeneous terms involving $u$ :

$$
\rho_{t t}-\Delta p=\nabla \cdot[\nabla \cdot(\rho u \otimes u)] .
$$

This is in fact what Sideris did in [13]. However, we will treat this equation in a very different manner from that in [13] due to the facts that $\rho(r) \rightarrow 0$ as $r \rightarrow \infty$ and the initial velocity field $u_{0}$ has no compact support. We will choose the modified Bessel function $K_{0}(r)$ in two-dimensional $(2 \mathrm{D})$ case and $\frac{1}{r e^{r}}$ in 3D case as test functions for the above quasi-linear wave type equation, respectively, to explore the nonlinear structure of the pressure $p$ as a function of $\rho$, which eventually corresponds to the formation of finite-time singularities. The second one is making use of the symmetric structure of the solutions, which results in a good sign for the inhomogeneous term $\nabla \cdot[\nabla \cdot(\rho u \otimes u)]$ when taking the inner product of the above wave-type equation with test functions. Moreover, the symmetric structure of the solutions will also be used to eliminate boundary terms on the artificial boundary $r=0$.

The remaining part of this paper is simply organized as follows: in Section 2, we will prove Theorem 1.1. Then in Section 3, we present the proof of Theorem 1.2.

\section{Singularities of compressible Euler equations in $3 D$}

In this section, we will prove Theorem 1.1. Before that, let us recall the local wellposedness of compressible Euler equations (for example, see [10]). To be precise, let us formulate it as a theorem for our use.

Theorem 2.1. Assume that $\gamma>1, \rho_{0} \geq 0$ and $\left(c_{0}, u_{0}\right) \in H^{3}\left(\mathbb{R}^{n}\right)$ with $c_{0}=c\left(\rho_{0}\right)$ and $n=2$, 3. Then there exists a unique solution $(\rho, u)$ to the compressible Euler equations (1.1) with initial data (1.3) on some time interval $[0, T)$, which satisfies

$$
c(\rho), u \in C\left([0, T), H^{3}\left(\mathbb{R}^{n}\right)\right) \cap C^{1}\left([0, T), H^{2}\left(\mathbb{R}^{n}\right)\right) \cap C^{2}\left([0, T), H^{1}\left(\mathbb{R}^{n}\right)\right),
$$

and

$$
\rho \in C\left([0, T) \times \mathbb{R}^{n}\right)
$$

If $1<\gamma \leq \frac{5}{3}$, then

$$
\rho \in C\left([0, T), H^{2}\left(\mathbb{R}^{n}\right)\right) \cap C^{1}\left([0, T), H^{1}\left(\mathbb{R}^{n}\right)\right) .
$$


If $\rho_{0}$ and $u_{0}$ are radially symmetric and (1.5) is satisfied, then

$$
\rho(t, 0) \equiv 0, u(t, 0) \equiv 0, \quad u(t, x)=\frac{x}{r} v(t, r) .
$$

The proof of the local well-posedness in the above theorem is based on the fact that the compressible Euler equations can be written as a symmetric hyperbolic system in terms of $(c, u)$ :

$$
\left\{\begin{array}{l}
c_{t}+u \cdot \nabla c+\frac{2 c}{\gamma-1} \nabla \cdot u=0 \\
u_{t}+u \cdot \nabla u+\frac{\gamma c}{\gamma-1} \nabla c=0
\end{array}\right.
$$

See [10] for more details. The fact (2.2) is due to Remark 1.3 and the equation of mass conservation in the original compressible Euler equations $(1.1)$. The fact $u(t, 0) \equiv 0$ is in fact a universal identity for smooth symmetric vector. To see $\rho(t, 0) \equiv 0$, we use $u(t, 0) \equiv 0$ and the equation of mass conservation to get

$$
\rho(t, 0)=\rho_{0}(0) e^{-\int_{0}^{t} \nabla \cdot u(s, 0) d s} .
$$

It is ready to present the proof of Theorem 1.1.

Proof. We prove Theorem 1.1 by contradiction. Suppose that the solution $(c, u) \in$ $H^{3}\left(\mathbb{R}^{3}\right)$ for all time $t \geq 0$ and $T=\infty$ in Theorem 2.1. We will derive that the density blows up in the ball centered at the origin with an arbitrary small radius $r_{0}>0$ in a finite time, which contradicts with (2.1).

Applying the time derivative to the first equation of the compressible Euler system (1.1), we have (in the sense of distribution)

$$
\rho_{t t}=-\nabla \cdot(\rho u)_{t}=\Delta p+\nabla \cdot[\nabla \cdot(\rho u \otimes u)] .
$$

Taking the $L^{2}$ inner product of the above equation with the test function $\frac{1}{r e^{r}}$, one has

$$
\frac{d^{2}}{d t^{2}} \int_{\mathbb{R}^{3}} \frac{\rho}{r e^{r}} d x=\int_{\mathbb{R}^{3}} \Delta p \frac{1}{r e^{r}} d x+\int_{\mathbb{R}^{3}} \nabla \cdot[\nabla \cdot(\rho u \otimes u)] \frac{1}{r e^{r}} d x .
$$

Let us first compute that

$$
\begin{aligned}
\int_{\mathbb{R}^{3}} & \Delta p \frac{1}{r e^{r}} d x \\
= & \int_{\mathbb{R}^{3}} p \Delta \frac{1}{r e^{r}} d x-\lim _{\varepsilon \rightarrow 0} \int_{|x|=\varepsilon}\left(\frac{\partial p}{\partial r} \frac{e^{-r}}{r}-\frac{d\left(\frac{1}{r e^{r}}\right)}{d r} p\right) d s \\
= & \int_{\mathbb{R}^{3}} p \frac{1}{r e^{r}} d x+\lim _{\varepsilon \rightarrow 0} \int_{|x|=\varepsilon}\left(\frac{1}{r e^{r}}\right)^{\prime} p d s .
\end{aligned}
$$

Using the equation of state $(1.2)$ and the fact that $\rho(t, 0) \equiv 0$, we have

$$
\begin{gathered}
A \int_{\mathbb{R}^{3}} \Delta \rho^{\gamma} \frac{1}{r e^{r}} d x=A \int_{\mathbb{R}^{3}} \rho^{\gamma} \frac{1}{r e^{r}} d x \\
\geq \frac{A}{(4 \pi)^{\gamma-1}}\left(\int_{\mathbb{R}^{3}} \frac{\rho}{r e^{r}} d x\right)^{\gamma} .
\end{gathered}
$$


On the other hand, noting (2.3), one has

$$
\begin{aligned}
\int_{\mathbb{R}^{3}} & \frac{1}{r e^{r}} \nabla \cdot[\nabla \cdot(\rho u \otimes u)] d x \\
= & \int_{\mathbb{R}^{3}} \partial_{i} \partial_{j}\left(\frac{1}{r e^{r}}\right)\left(\rho u_{i} u_{j}\right) d x \\
= & \int_{\mathbb{R}^{3}} \rho v^{2} \frac{x_{j}}{r} \partial_{r}\left[\frac{x_{j}}{r}\left(\frac{1}{r e^{r}}\right)^{\prime}\right] d x \\
= & \int_{\mathbb{R}^{3}} \rho v^{2}\left(\frac{1}{r e^{r}}\right)^{\prime \prime} d x \\
= & \int_{\mathbb{R}^{3}} \rho v^{2}\left[\frac{1}{r}+\frac{2}{r^{2}}+\frac{2}{r^{3}}\right] e^{-r} d x>0 .
\end{aligned}
$$

Here and in what follows, we use Einstein's convention for summation over repeated indices. Consequently,

$$
\frac{d^{2}}{d t^{2}} F(t) \geq \frac{A}{(4 \pi)^{\gamma-1}}(F(t))^{\gamma}
$$

where

$$
F(t)=\int_{\mathbb{R}^{3}} \frac{\rho(t, r)}{r e^{r}} d x .
$$

Using the equation of conservation of mass and integration by parts, and noting (1.7), one has

$$
F^{\prime}(0)=\left.\frac{d}{d t} \int_{\mathbb{R}^{3}} \frac{\rho}{r e^{r}} d x\right|_{t=0}=-\int_{\mathbb{R}^{3}} \frac{(1+r) \rho_{0} v_{0}}{r^{2} e^{r}} d x>0 .
$$

The combination of (2.5) and (2.6) gives that

$$
F^{\prime}(t)=F^{\prime}(0)+\int_{0}^{t} \frac{d^{2}}{d s^{2}} F(s) d s \geq F^{\prime}(0)>0 .
$$

Consequently, one can multiply the both sides of (2.5) by $\frac{d}{d t} F(t)$ to get

$$
\begin{aligned}
\left(F^{\prime}(t)\right)^{2} \geq & \frac{A}{(\gamma+1)(4 \pi)^{\gamma-1}}(F(t))^{\gamma+1} \\
& +\left.\left(\frac{d}{d t} \int_{\mathbb{R}^{3}} \frac{\rho}{r e^{r}} d x\right)^{2}\right|_{t=0}-\frac{A}{(\gamma+1)(4 \pi)^{\gamma-1}}\left(\int_{\mathbb{R}^{3}} \frac{\rho_{0}}{r e^{r}} d x\right)^{\gamma+1} .
\end{aligned}
$$

Noting (1.7) and (2.6), and by denoting

$$
C_{0}=\sqrt{\frac{A}{(\gamma+1)(4 \pi)^{\gamma-1}}},
$$

one has

which implies that

$$
F^{\prime}(t) \geq C_{0}(F(t))^{\frac{\gamma+1}{2}}
$$

$$
\int_{\mathbb{R}^{3}} \frac{\rho}{r e^{r}} d x \geq\left(F(0)^{-\frac{\gamma-1}{2}}-\frac{\gamma-1}{2} C_{0} t\right)^{-\frac{2}{\gamma-1}} .
$$


Noting (1.6), one has

$$
\int_{\mathbb{R}^{3}} \frac{\rho_{0}}{r e^{r}} d x>0
$$

By using the mass conservation, one has

$$
F(t)=\int_{\mathbb{R}^{3}} \frac{\rho}{r e^{r}} d x \leq \int_{B_{r_{0}}} \frac{\rho}{r e^{r}} d x+\frac{1}{r_{0}} \int_{\mathbb{R}^{3}} \rho_{0} d x,
$$

for any given $r_{0}>0$, here $B_{r_{0}}$ is the $3 \mathrm{D}$ ball centered at the origin with radius $r_{0}$. Consequently, one concludes that $\int_{r \leq r_{0}} \rho(t, r) r d r$ cannot be bounded as $t \rightarrow$ $\frac{2 F(0)^{-\frac{\gamma-1}{2}}}{(\gamma-1) C_{0}}$, which in turn implies that $\rho(t, r)$ will blow up for $r \leq r_{0}$ as $t \rightarrow \frac{2 F(0)^{-\frac{\gamma-1}{2}}}{(\gamma-1) C_{0}}$. By the assumption $c \in L^{\infty}\left([0, \infty), H^{3}\left(\mathbb{R}^{3}\right)\right.$ and Sobolev imbedding theorem, one has $c \in L^{\infty}\left([0, \infty) \times \mathbb{R}^{3}\right)$ and hence $\rho \in L^{\infty}\left([0, \infty) \times \mathbb{R}^{3}\right)$. We arrive at a contradiction. So, the $H^{3}$ norm of $(c, u)$ cannot be bounded before the time $\frac{2 F(0)^{-\frac{\gamma-1}{2}}}{(\gamma-1) C_{0}}$. The proof of Theorem 1.1 is completed.

\section{Singularities of compressible Euler equations in $2 \mathrm{D}$}

In this section, we prove Theorem 1.2. First of all, let us recall that the modified Bessel function $K_{0}(r)$ and its derivative $K_{0}^{\prime}(r)$ decay sufficiently fast as $r \rightarrow \infty$. In fact, for any given $k>1$, one has

$$
\begin{aligned}
\sup _{0<r<\infty} r^{k} K_{0}(r)= & \sup _{0<r<\infty} r^{k} \int_{0}^{\infty} e^{-r \cosh t} d t \\
= & \sup _{0<r<\infty} r^{k} \int_{0}^{1} e^{-\frac{r e t}{2}} e^{-\frac{r e-t}{2}} d t+\sup _{0<r<\infty} r^{k} \int_{1}^{\infty} e^{-\frac{r e t}{2}} e^{-\frac{r e-t}{2}} d t \\
\leq & \sup _{0<r<\infty} r^{k} e^{-\frac{r}{2 e}} \int_{0}^{1} e^{-\frac{r e^{t}}{2}} d t \\
& +\sup _{0<r<\infty} \int_{1}^{\infty} r^{k} e^{-\frac{r e^{t}}{2}}\left(\frac{r}{2} e^{t}\right)^{k}\left(\frac{2}{r} e^{-t}\right)^{k} d t \\
\leq & \sup _{0<r<\infty} r^{k} e^{-\frac{r}{2 e}}+\sup _{0<r<\infty} 2^{k} \int_{1}^{\infty} e^{-k t} d t \sup _{0<s<\infty} e^{-s} s^{k} \\
<\infty &
\end{aligned}
$$

and

$$
\begin{aligned}
\sup _{0<r<\infty} r^{k}\left|K_{0}^{\prime}(r)\right| & =\sup _{0<r<\infty} r^{k} \int_{0}^{\infty} e^{-r \cosh t} \cosh t d t \\
& =\sup _{0<r<\infty} 2 r^{k} \int_{0}^{1} e^{-\frac{r e}{2}} e^{-\frac{r e^{-t}}{2}} d t+\sup _{0<r<\infty} r^{k} \int_{1}^{\infty} e^{-\frac{r e t}{2}} e^{-\frac{r e^{-t}}{2}} e^{t} d t \\
& \leq \sup _{0<r<\infty} 2 r^{k} e^{-\frac{r}{2 e}}+\sup _{0<r<\infty} 2^{k} \int_{1}^{\infty} e^{-(k-1) t} d t \sup _{0<s<\infty} e^{-s} s^{k} \\
& <\infty
\end{aligned}
$$


On the other hand, for $r>0$, we also have

$$
\begin{aligned}
K_{0}(r) & \leq \int_{0}^{1} e^{-r \cosh t} d t+\int_{1}^{\infty} e^{-\frac{r e t}{2}} e^{-\frac{r e^{-t}}{2}} d t \\
& \leq 1+\int_{1}^{\infty} e^{-\frac{r e^{t}}{2}} d t \leq 1+\frac{2}{r},
\end{aligned}
$$

and

$$
\begin{aligned}
\left|K_{0}^{\prime}(r)\right| & \leq \int_{0}^{1} e^{-r \cosh t} e^{t} d t+\int_{1}^{\infty} e^{-\frac{r e e^{t}}{2}} e^{-\frac{r e^{-t}}{2}} e^{t} d t \\
& \leq 2+\int_{1}^{\infty} e^{-\frac{r e e^{t}}{2}} e^{t} d t \\
& \leq 2+\int_{1}^{\infty} 2\left(\frac{r e^{t}}{2}\right)^{-2} e^{t} d t \leq 2+\frac{1}{2 r^{2}} .
\end{aligned}
$$

We in fact have proved the following lemma:

Lemma 3.1. The modified Bessel function $K_{0}(r)=\int_{0}^{\infty} e^{-r \cosh t} d t$ satisfies

$$
\left\{\begin{array}{l}
K_{0}(r) \leq \frac{3}{r},\left|K_{0}^{\prime}(r)\right| \leq \frac{1}{r^{2}}, \quad 0<r<\frac{1}{2}, \\
K_{0}(r) \leq \frac{C_{k}}{r^{k}},\left|K_{0}^{\prime}(r)\right| \leq \frac{C_{k}}{r^{k}}, \quad r>1,
\end{array}\right.
$$

for constants $C_{k}$ depending only on $k>1$.

Now it is ready to present the proof of Theorem 1.2.

Proof. We prove Theorem 1.2 by contradiction. Suppose that the solution $(c, u) \in$ $H^{3}\left(\mathbb{R}^{3}\right)$ for all time $t \geq 0$ and $T=\infty$ in Theorem 2.1. We will derive that the density blows up in the ball centered at the origin with an arbitrary small radius $r_{0}>0$ in a finite time, which contradicts with (2.1).

Taking the $L^{2}$ inner product of the above equation with the test function $K_{0}(r)$, one has

$$
\frac{d^{2}}{d t^{2}} \int_{\mathbb{R}^{2}} \rho K_{0}(r) d x=\int_{\mathbb{R}^{2}} \Delta p K_{0}(r) d x+\int_{\mathbb{R}^{2}} \nabla \cdot[\nabla \cdot(\rho u \otimes u)] K_{0}(r) d x .
$$

Using a similar argument as in Section 2 and noting the decay properties of the Bessel function $K_{0}(r)$ in Lemma 3.1, we have

$$
\int_{\mathbb{R}^{2}} \Delta p K_{0}(r) d x=\int_{\mathbb{R}^{2}} p \Delta K_{0}(r) d x
$$

Noting that the modified Bessel function $K_{0}(r)$ satisfies

$$
K_{0}^{\prime \prime}+\frac{1}{r} K_{0}^{\prime}=K_{0}
$$

one has

$$
\begin{aligned}
\int_{\mathbb{R}^{2}} \Delta p K_{0}(r) d x & =A \int_{\mathbb{R}^{2}} \rho^{\gamma} K_{0}(r) d x \\
& \geq \frac{A}{\left(\int_{\mathbb{R}^{2}} K_{0}(r) d x\right)^{\gamma-1}}\left(\int_{\mathbb{R}^{2}} \rho K_{0}(r) d x\right)^{\gamma} .
\end{aligned}
$$


On the other hand, noting (2.3) and using (3.1) and Lemma 3.1, one has

$$
\begin{aligned}
\int_{\mathbb{R}^{2}} & K_{0}(r) \nabla \cdot[\nabla \cdot(\rho u \otimes u)] d x \\
\quad= & \int_{\mathbb{R}^{2}} \rho v^{2} K_{0}^{\prime \prime}(r) d x \\
= & \int_{\mathbb{R}^{2}} \rho v^{2}\left(K_{0}(r)-\frac{1}{r} K_{0}^{\prime}(r)\right) d x>0 .
\end{aligned}
$$

Here, we also used the fact that $K_{0}^{\prime}(r)<0$ by the expression of the modified Bessel function $K_{0}(r)$. Consequently, we have

$$
\frac{d^{2}}{d t^{2}} G(t) \geq \frac{A G(t)^{\gamma}}{\left(\int_{\mathbb{R}^{2}} K_{0}(r) d x\right)^{\gamma-1}} .
$$

where

$$
G(t)=\int_{\mathbb{R}^{2}} \rho(t, r) K_{0}(r) d x .
$$

Using the equation of conservation of mass and integration by parts, and noting (1.9), one has

$$
G^{\prime}(0)=\left.\frac{d}{d t} \int_{\mathbb{R}^{2}} \rho K_{0}(r) d x\right|_{t=0}=\int_{\mathbb{R}^{2}} \rho_{0} v_{0} K_{0}^{\prime}(r) d x>0 .
$$

The combination of (3.2) and (3.3) gives that

$$
G^{\prime}(t)=G^{\prime}(0)+\int_{0}^{t} \frac{d^{2}}{d s^{2}} G(s) d s \geq G^{\prime}(0)>0 .
$$

Consequently, one can multiply the both sides of (3.2) by $\frac{d}{d t} G(t)$ to get

$$
\begin{aligned}
\left(G^{\prime}(t)\right)^{2} \geq & \frac{A}{(\gamma+1)\left(\int_{\mathbb{R}^{2}} K_{0}(r) d x\right)^{\gamma-1}}(G(t))^{\gamma+1} \\
& +\left.\left(\frac{d}{d t} \int_{\mathbb{R}^{2}} \rho K_{0}(r) d x\right)^{2}\right|_{t=0}-\frac{A\left(\int_{\mathbb{R}^{2}} \rho_{0} K_{0}(r) d x\right)^{\gamma+1}}{(\gamma+1)\left(\int_{\mathbb{R}^{2}} K_{0}(r) d x\right)^{\gamma-1}}
\end{aligned}
$$

Noting (1.10) and (3.3), and by denoting

$$
C_{1}=\sqrt{\frac{A}{(\gamma+1)}}\left(\int_{\mathbb{R}^{2}} K_{0}(r) d x\right)^{-\frac{\gamma-1}{2}},
$$

one has

$$
G^{\prime}(t) \geq C_{1}(G(t))^{\frac{\gamma+1}{2}}
$$

which implies that

$$
\int_{\mathbb{R}^{2}} \rho K_{0}(r) d x \geq\left(G(0)^{-\frac{\gamma-1}{2}}-\frac{\gamma-1}{2} C_{1} t\right)^{-\frac{2}{\gamma-1}} .
$$

Noting (1.9), one has

$$
G(0)=\int_{\mathbb{R}^{2}} \rho_{0} K_{0}(r) d x>0
$$


By using the mass conservation, one has

$$
G(t)=\int_{\mathbb{R}^{2}} \frac{\rho}{r e^{r}} d x \leq \int_{B_{r_{0}}} \rho K_{0}(r) d x+\frac{\max _{r \geq r_{0}} K_{0}(r)}{r_{0}} \int_{\mathbb{R}^{2}} \rho_{0} d x,
$$

for any given $r_{0}>0$, here $B_{r_{0}}$ is the $2 \mathrm{D}$ ball centered at the origin with radius $r_{0}$. Consequently, one concludes that $\rho(t, r)$ will blow up for $r \leq r_{0}$ as $t \rightarrow \frac{2 G(0)^{-\frac{\gamma-1}{2}}}{(\gamma-1) C_{1}}$. By the assumption $c \in L^{\infty}\left([0, \infty), H^{3}\left(\mathbb{R}^{2}\right)\right.$ and Sobolev imbedding theorem, one has $c \in L^{\infty}\left([0, \infty) \times \mathbb{R}^{2}\right)$ and hence $\rho \in L^{\infty}\left([0, \infty) \times \mathbb{R}^{2}\right)$. We arrive at a contradiction. So, the $H^{3}$ norm of $(c, u)$ cannot be bounded before the time $\frac{2 G(0)^{-\frac{\gamma-1}{2}}}{(\gamma-1) C_{1}}$. The proof of Theorem 1.2 is completed.

\section{Acknowledgment}

This work was done when Zhen Lei and Yi Du were visiting the Department of Mathematics of Penn State University during 2012. They would like to thank professor Chun Liu, professor Qiang Du and the institute for their hospitality. Yi Du was supported by the NSFC (grant no. 11001088) and Pearl River New Star (grant no. 2012J2200016). Zhen Lei was supported by NSFC (grants numbers 11171072 and 11222107), the Foundation for Innovative Research Groups of NSFC (grant no. 11121101), FANEDD, NCET-12-0120, Innovation Program of Shanghai Municipal Education Commission (grant no. 12ZZ012), SGST(grant no. 09DZ2272900) and ShangHai Rising Star program (10QA1400300). This work was also supported by the NSF (grant: DMS-1109107) of Professor Chun Liu.

\section{References}

[1] C. Bardos and E.S. Titi, Euler equations for incompressible ideal fluids, Usp. Mat. Nauk 62(3) (2007), 5-46.

[2] A. Castro, D. Cordoba, C. Fefferman, F. Gancedo and M. Gomez-Serrano, Finite time singularities for the free boundary incompressible Euler equations, (2011), arXiv:1112.2170v1.

[3] P. Constantin, Note on loss of regularity for solutions of the 3D incompressible Euler and related equations, Commun. Math. Phys. 104 (1986), 311-326.

[4] P. Constantin, On the Euler equations of incompressible fluids, Bull. Amer. Math. Soc., 44 (2007), 603-621.

[5] P. Constantin, C. Fefferman and A. Majda, Geometric constraints on potentially singular solutions for the 3-D Euler equation, Comm. PDE. 21 (1996), 559-571.

[6] D. Cordoba and C. Fefferman, On the collapse of tubes carried by 3D incompressible flows, Comm. Math. Phys. 222 (2001), 293-298.

[7] D. Coutand and S. Shkoller, On the finite-time splash and splat singularities for the 3-D freesurface Euler equations, (2012), avaliable online at arXiv:1201.4919v2.

[8] T.Y. Hou and L. Zhen, On the stabilizing effect of convection for three dimensional incompressible flows, Comm. Pure Appl. Math. 62(4) (2009), 501-564.

[9] J. Jang and N. Masmoudi, Well-posedness of compressible Euler equations in a physical vacuum, arXiv.org/pdf/1005.4441.

[10] T. Kato, The Cauchy problem of the quasi-linear symmetric hyperbolic systems, Arch. Ration. Mech. Anal. 58 (1975), 181-205.

[11] Z. Lei, Blow-up of classical solutions to the ideal magnetohydrodynamic equations with initial disturbances, (in Chinese) Chin. Ann. Math. Ser. A 26(2) (2005), 193-204. (Translation in Chin. J. Contemp. Math. 26(2) (2005),157-168.)

[12] P.L. Lions, Mathematical topics in fluid mechanics: compressible models, Oxford Lecture Series in Mathematics and Its Applications, 2 (1998), no. 10, Oxford University Press. 
[13] T.C. Sideris, Formation of singularities in three-dimensional compressible fluids, Comm. Math. Phys. 101 (1985), 475-485.

School of Mathematical Sciences, LMns and Shanghai Key Laboratory for Contemporary Applied Mathematics, Fudan University, Shanghai 200433, People's RePublic OF CHINA

E-mail address: leizhn@gmail.com

School of Mathematical Sciences, South China Normal University, Guangzhou 510631, People's Republic of China

E-mail address: duyidy@gmail.com

Department of Mathematics, Penn State University, PA 16801, USA

E-mail address: zhang_q@math.psu.edu. 